\title{
Scanning and transmission electron microscopic studies of human intestinal mucosa
}

\author{
PETER G. TONER, KATHARINE E. CARR ${ }^{1}$, ANNE FERGUSON, AND \\ COLIN MACKAY \\ From the Departments of Pathology and Surgery, University of Glasgow Western Infirmary, Glasgow, \\ the Department of Medicine, Royal Infirmary Glasgow, and the Bio-Engineering Unit, University of \\ Strathclyde, Glasgow
}

SUMMARY The scanning electron microscope is capable of $25 \mathrm{~m} \mu$ resolution combined with a great depth of focus, features which make the technique of value in the study of the intestinal mucosa. Surgical biopsies and postmortem specimens of small intestine have been examined using scanning and transmission electron microscopy. In biopsies, at low magnification, the villous pattern is seen while at higher magnification details of cell surfaces may be observed. Some features are best observed using scanning microscopy to examine dewaxed thick histological sections. In necropsy specimens villous architecture can still be satisfactorily assessed and details of the villous cores are seen, their collagen skeletons apparently less robust than the villi of the fresh biopsy. Scanning microscopy can extend the three-dimensional study of the small intestinal mucosa beyond the limits imposed by the resolution of the dissecting microscope.

The technique of peroral intestinal biopsy introduced by Shiner (1956 and 1957) has made available fresh human tissues for examination not only by conventional histological and ultrastructural methods but also by binocular dissecting light microscopy (Rubin and Dobbins, 1965; Trier and Rubin, 1965). Two further methods have recently been applied to the investigation of intestinal morphology. First, Loehry and Creamer (1966) have shown that the pattern of the mucosa is retained, despite autolysis, in specimens obtained at necropsy. A surprisingly detailed assessment of mucosal architecture is thus possible using the dissecting microscope (Creamer and Leppard, 1965; Ferguson, Maxwell, and Carr, 1969; Loehry and Creamer, 1969). Second the range of dissecting light microscopy has been extended by the use of the scanning electron microscope, allowing examination of the surface contours of individual villi as well as the overall pattern of the mucosa (Carr and Toner, 1968; Marsh, Swift, and Williams, 1968; Toner and Carr, 1969; Demling,

Received for publication 22 October 1969.

'Present address: Department of Anatomy, University of Glasgow.
Becker, and Classen, 1969). The present paper describes further applications of scanning electron microscopy to the study of human intestine, including its use in the examination of postmortem specimens and of thick sections.

\section{Material and Methods}

The preparation of intestinal biopsies for scanning electron microscopy has been described in previous papers (Carr and Toner, 1968; Toner and Carr, 1969). Specimens of human jejunal and duodenal mucosa were obtained at laparotomy during the surgical treatment of patients with duodenal ulcers. Twenty-five such samples have now been examined as a basis for this study. In addition, specimens of small bowel obtained at necropsy were stored at room temperature for several days in saline, allowing further autolysis. They were then fixed in formalin and examined by dissecting microscopy (Ferguson et al, 1969). Pieces of tissue up to $10 \mathrm{~mm}$ wide were then washed, postfixed in osmium tetroxide, and pre- 


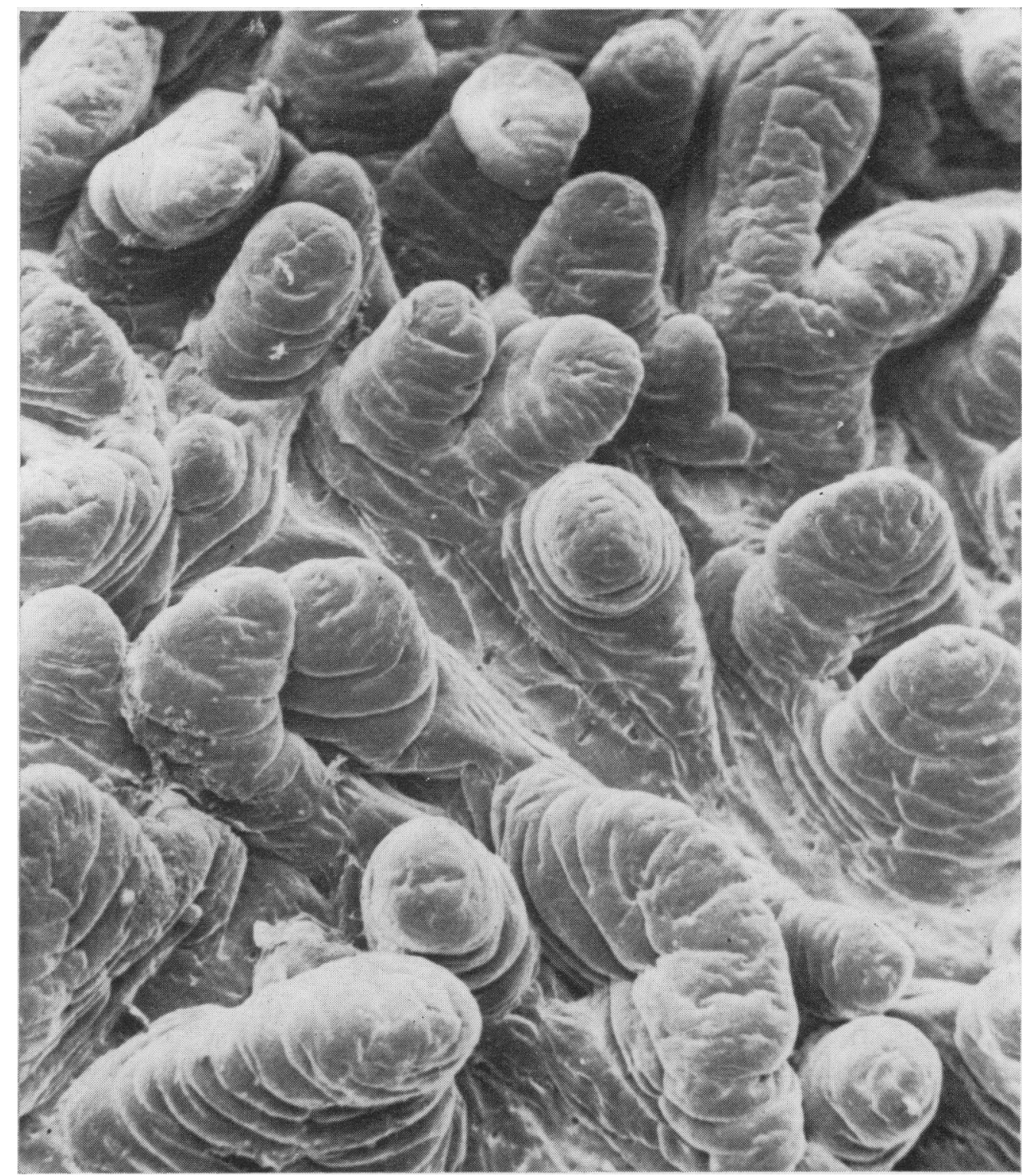

Fig. 1 Low magnification scanning electron micrograph of human jejunal mucosa obtained at surgical biopsy. The majority of villi are fingershaped, but occasional bifid and leaf-shaped villi can be easily recognized. The characteristic resolution and depth of focus are seen. $\times 100$.

pared for scanning electron microscopy in the same way as fresh specimens.

Blocks were also prepared for conventional histology. Sections $30 \mu$ thick were cut from these blocks and were mounted and prepared for scanning electron microscopy. ${ }^{1}$ After dewaxing and drying, these sections were coated with carbon-platinum and gold-palladium or with gold-palladium alone, in a vacuum evaporator.

${ }^{1}$ The scanning microscope used was the Cambridge Stereoscan Mk 2a, operated at accelerating voltages of from 2 to $30 \mathrm{kv}$.
Parallel investigations were also undertaken using transmission electron microscopy. Specimens postfixed in osmium tetroxide were embedded in Araldite, then sectioned and examined in the Siemens Elmiskop 1a.

\section{Results}

SCANNING ELECTRON MICROSCOPY OF INTESTINAL BIOPSIES

A typical low magnification scanning electron micrograph of the mucosal surface of human jejunum is shown in Fig. 1, which displays the resolution and depth of focus which are characteristic of the scanning image. The features seen in these specimens (Carr and Toner, 1968; Marsh 


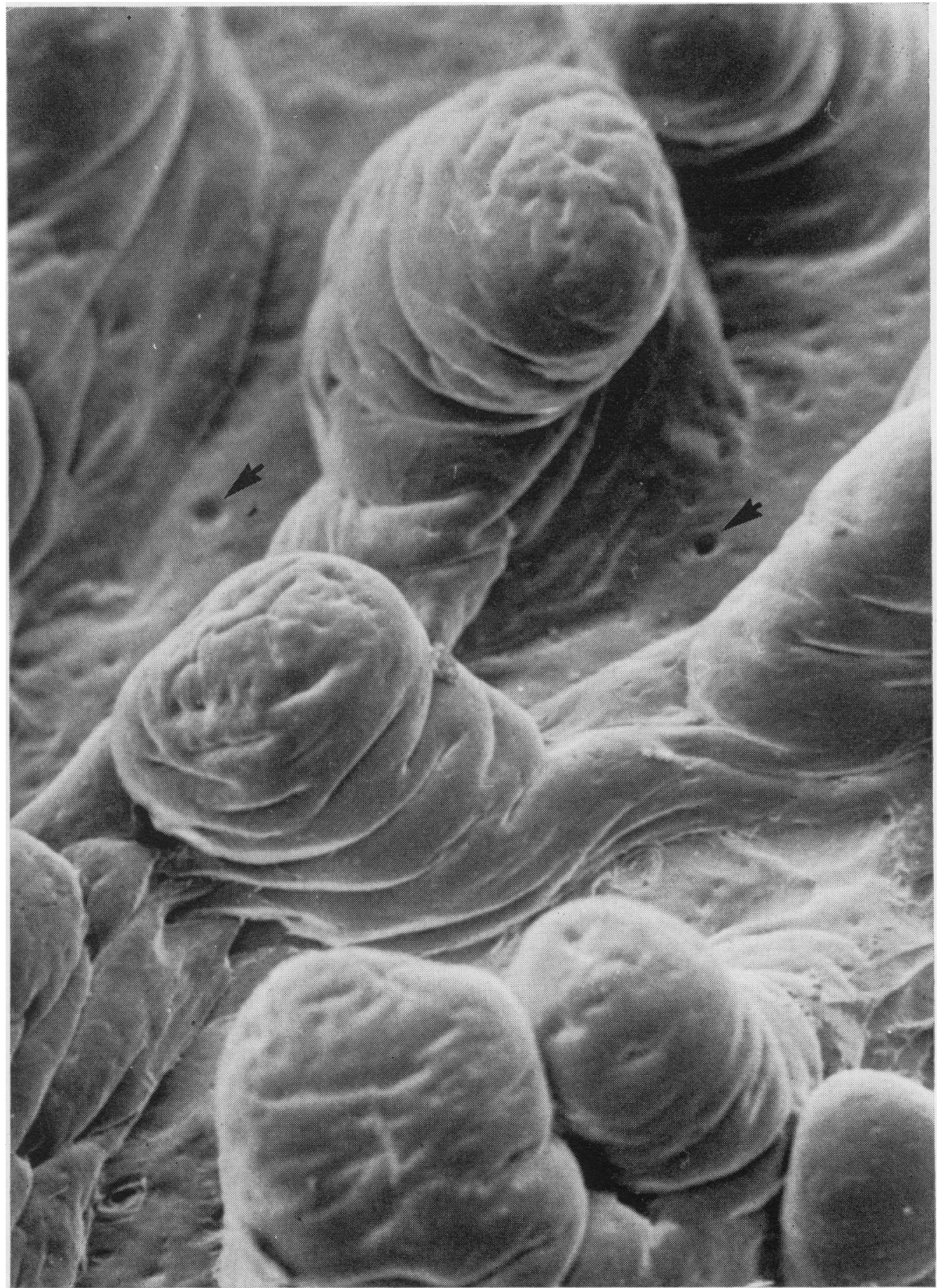

Fig. 2 Slightly higher magnification scanning electron micrograph of human jejunal mucosa showing the surface creases of the villi and occasional crypt mouths (arrows). $\times 260$. 
et al, 1968; Toner and Carr, 1969), include surface creases on villi, goblet cell mouths, and occasional intestinal crypt mouths opening between villi (Figs. 1 and 2). At high magnification (Marsh et al, 1968; Toner and Carr, 1969) hexagonal patterns formed by the close-packed apical surfaces of the columnar cells may be seen. In the fixed and air-dried biopsies examined, microvilli are not readily demonstrated although they have been seen in freeze-dried specimens (Marsh et al, 1968).

Dewaxed paraffin sections, $30 \mu$, of intestinal biopsies show some aspects of internal tissue structure which cannot be visualized in whole mucosal specimens. Among the features observed in these sections are the interconnecting intercellular spaces between the columnar cell bases (Figs. 3 and 4). At the free border of the epithelial cells there is a zone which corresponds to the striated border (Fig. 5). This can be resolved into its component microvilli when cut in longitudinal section. Limited areas of the surfaces of the villi are still available for examination in $30 \mu$ sections; a simultaneous display of the cut surface of the section and, at right angles, the natural free surface of the villus, is easily achieved (Fig. 3). In these cases, in addition to the surface features already described, the general pattern of arrangement of the microvilli can now often be seen (Fig. 6), due perhaps to minor structural alterations produced by wax embedding. Large intracellular features can also be observed in thick sections. Paneth cell granules, for example, are clearly seen at the bases of intestinal crypts (Fig. 7). Within the core of the villus and in the lamina propria, blood vessels are recognized by their contained red blood corpuscles (Fig. 8) and the connective tissue framework of collagen fibre bundles can be seen.

\section{SCANNING ELECTRON MICROSCOPY OF NECROPSY SPECIMENS}

The mucosal surface of autolysed small intestine, which retains the general structural features present at death, is recorded with clarity using the scanning electron microscope (Fig. 9). As in biopsies examined at low magnification, individual villi of different shapes can be identified, but in autolysed tissues the villous cores are more angular and often appear twisted. In some cases the villi may even appear collapsed or flattened, but are still easily recognizable. Figure 10 demonstrates the appearance of the connective tissue 'skeleton' of the mucosa in a case found to have a 'flat' intestinal mucosa. The absence of villi emphasizes the width of the crypt mouths which are much more prominent than in biopsy specimens owing to shedding of the autolysed crypt cells. In specimens with a more normal villous pattern the relationship between crypts and villi can be seen.

The surface of autolysed villi is surprisingly

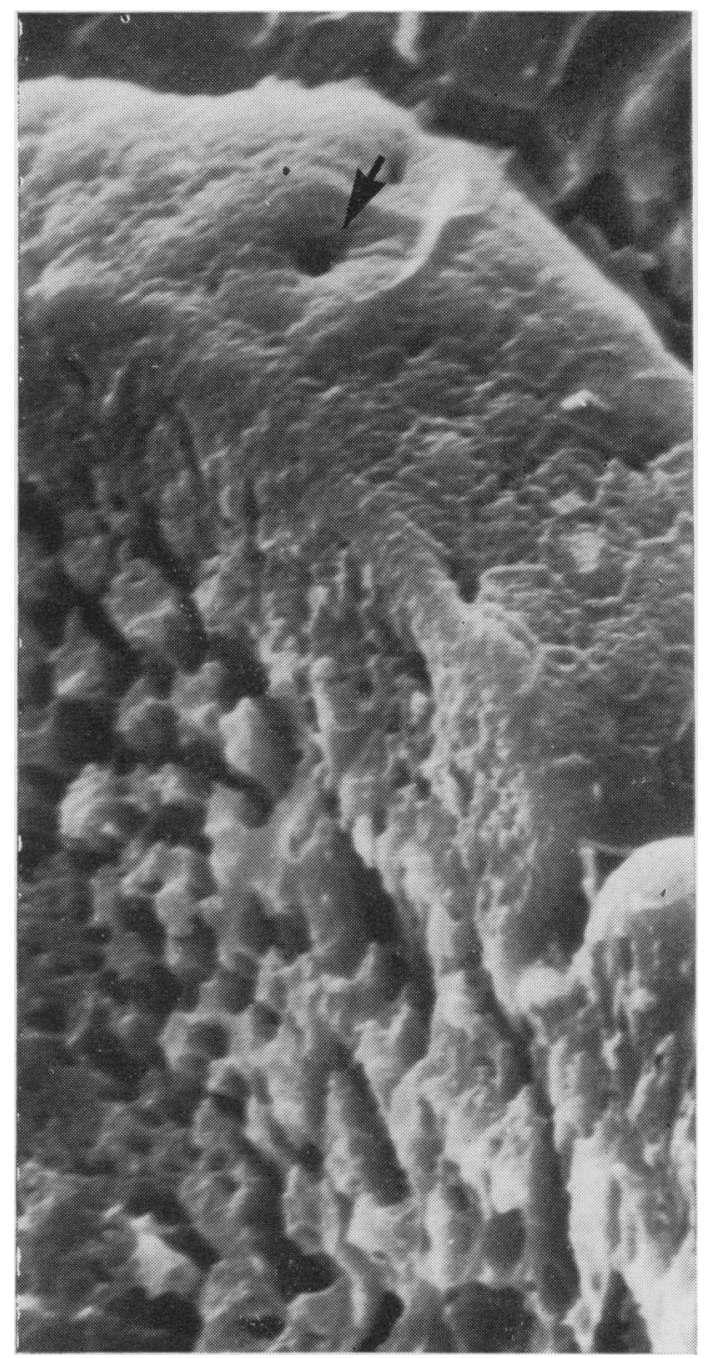

Fig. 3 Scanning electron micrograph of dewaxed $30 \mu$ thick section of human jejunum showing both the cut surface and the natural free surface of the villus. Intercellular spaces are seen between the lower parts of the epithelial cells on the cut surface, while the hexagonal pattern of adjacent cell boundaries can be made out on the free surface. A pit corresponding to a goblet cell mouth is present (arrow). $\times 1,750$.

Fig. 4 Scanning electron micrograph of dewaxed $30 \mu$ thick section of human jejunum showing the epithelial cells cut slightly obliquely, revealing the intercellular spaces. The 'basement membrane' region of the villus is displayed $(B) . \times 1,925$.

Fig. 5 Scanning electron micrograph of dewaxed $30 \mu$ thick section of human jejunum showing the apical parts of the epithelium of two adjacent villi. Although little intracellular detail is seen, the region of the striated border is readily seen (arrows). $A$ 'microcrypt' is present $(C)$, corresponding to the grooves seen in Figs. 1 and 2 in the whole specimen. $\times 2,500$. 


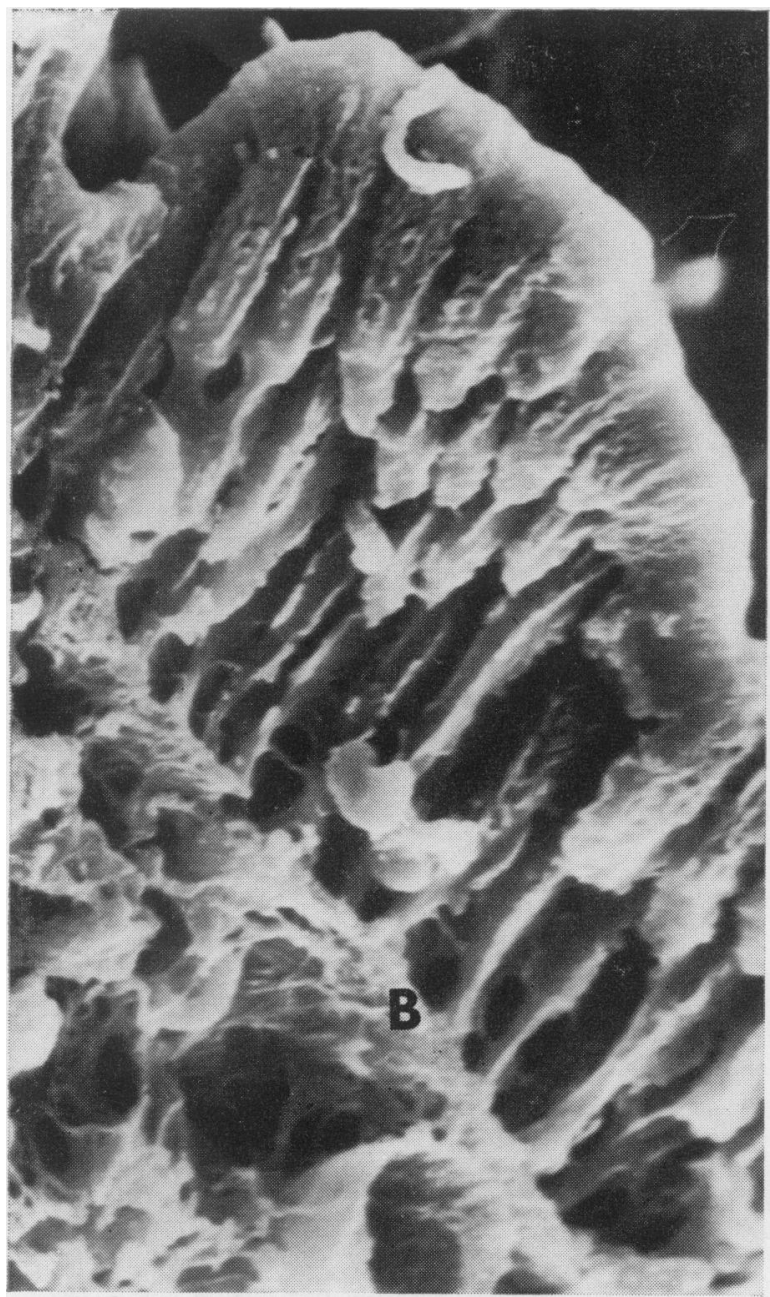

Fig. 4.

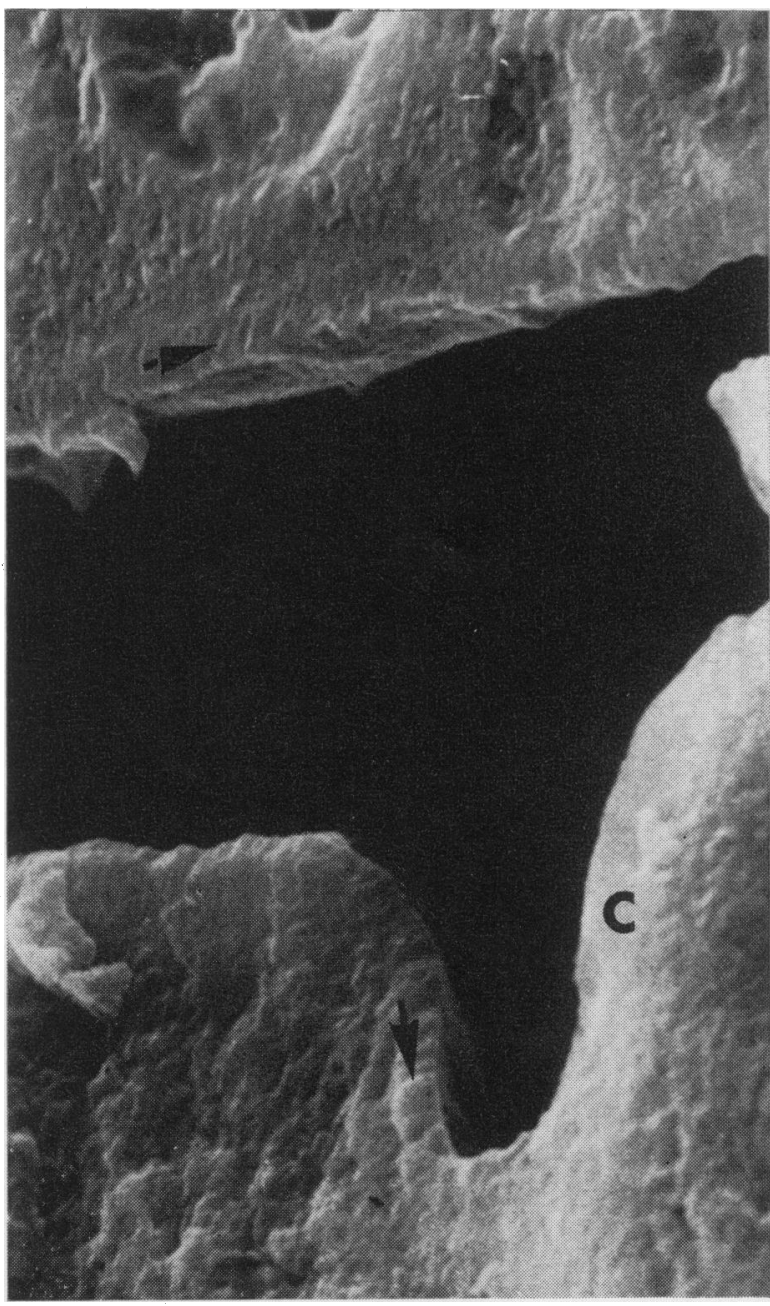

Fig. 5.

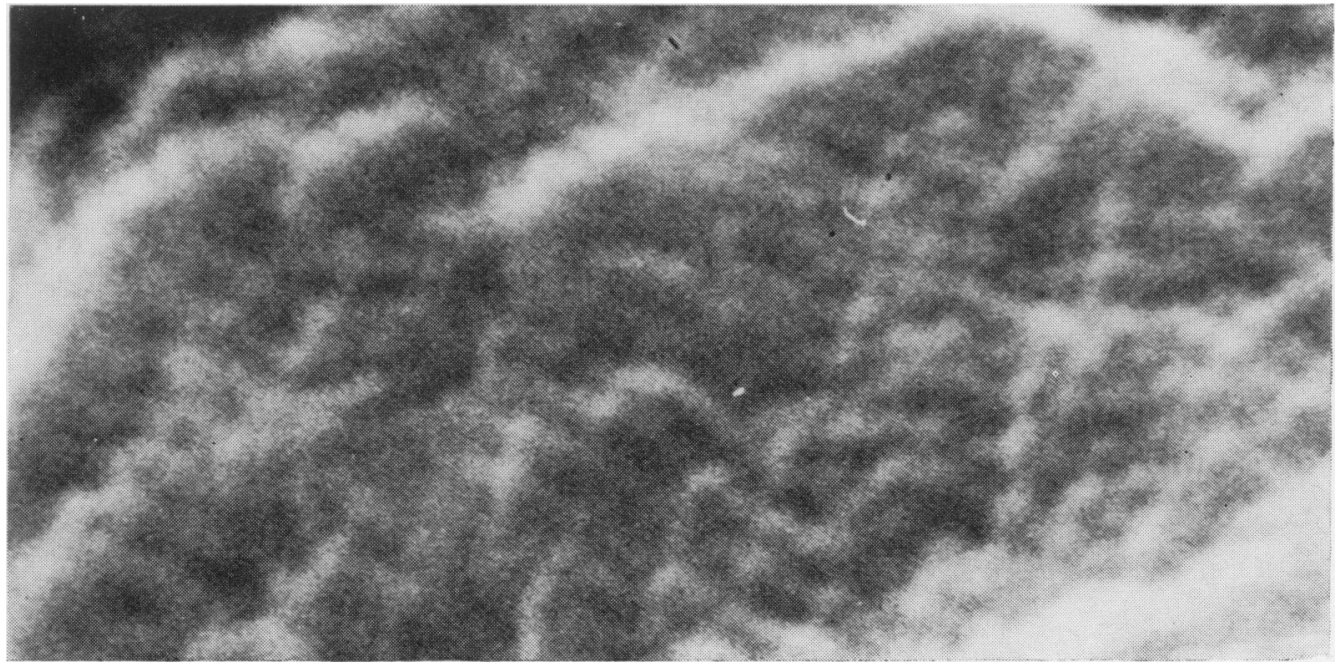

Fig. 6 Scanning electron micrograph of microvilli in the columnar cell surface of a human jejunal villus. The material was a dewaxed $30 \mu$ section. Only the surface pattern of arrangement of these microvilli can be seen. $\times 40,000$. 


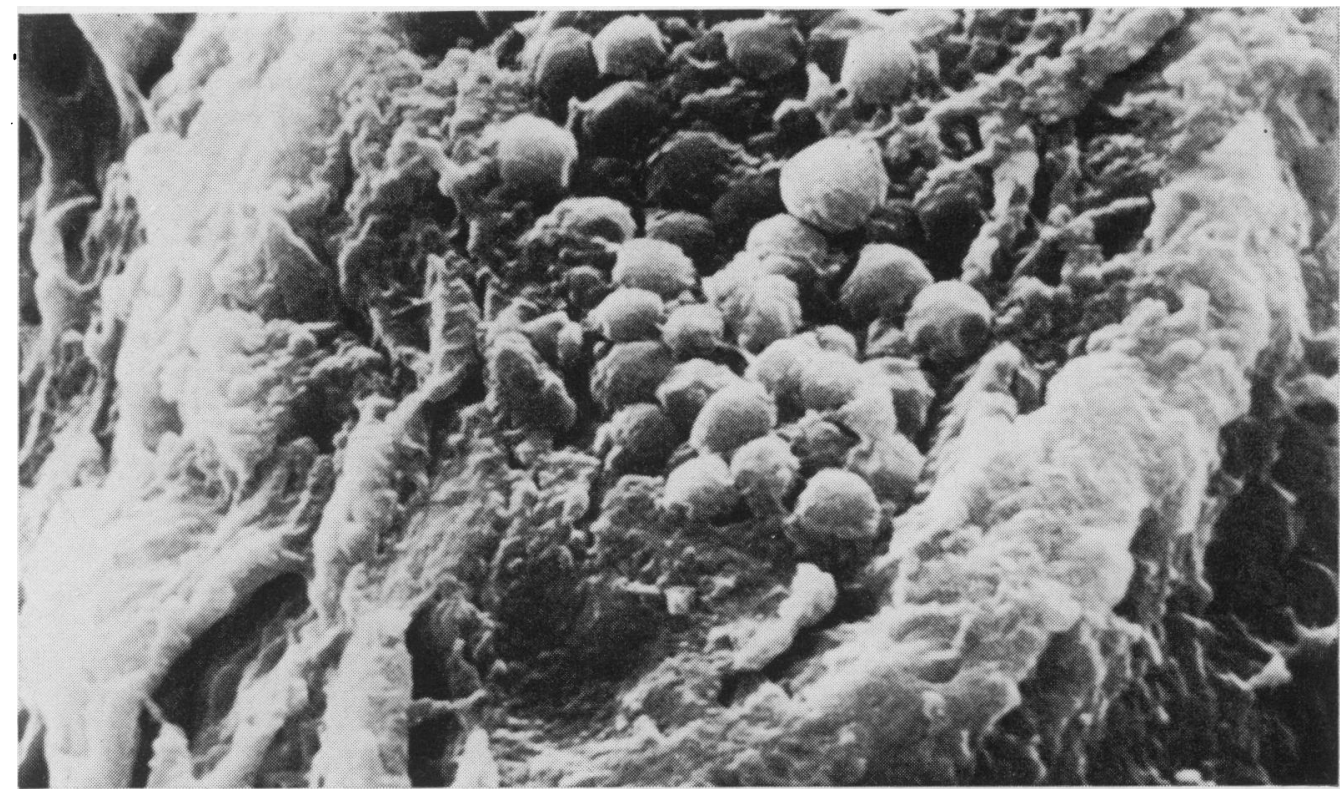

Fig. 7 Scanning electron micrograph of a dewaxed $30 \mu$ section of human jejunum showing part of the base of a crypt. The round structures are Paneth cell granules, but other intracellular details cannot be clearly distinguished. $\times 4,000$.

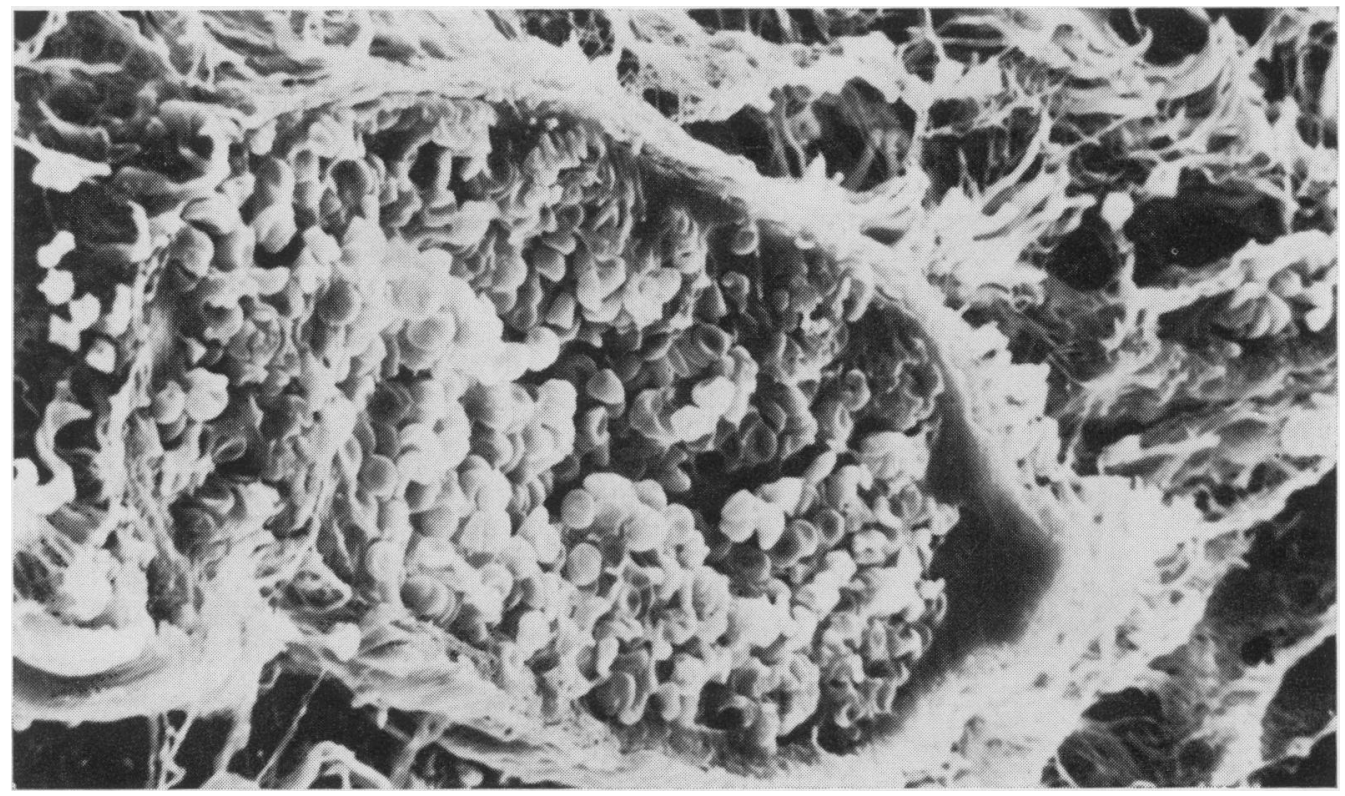

Fig. 8 Scanning electron micrograph of a blood vessel, probably a venule, in the lamina propria of the human jejunum, seen in a dewaxed $30 \mu$ section. The red blood cells are readily identified. Collagen fibre bundles surrounding the vessel and in the adjacent connective tissue are seen. $\times 750$. 


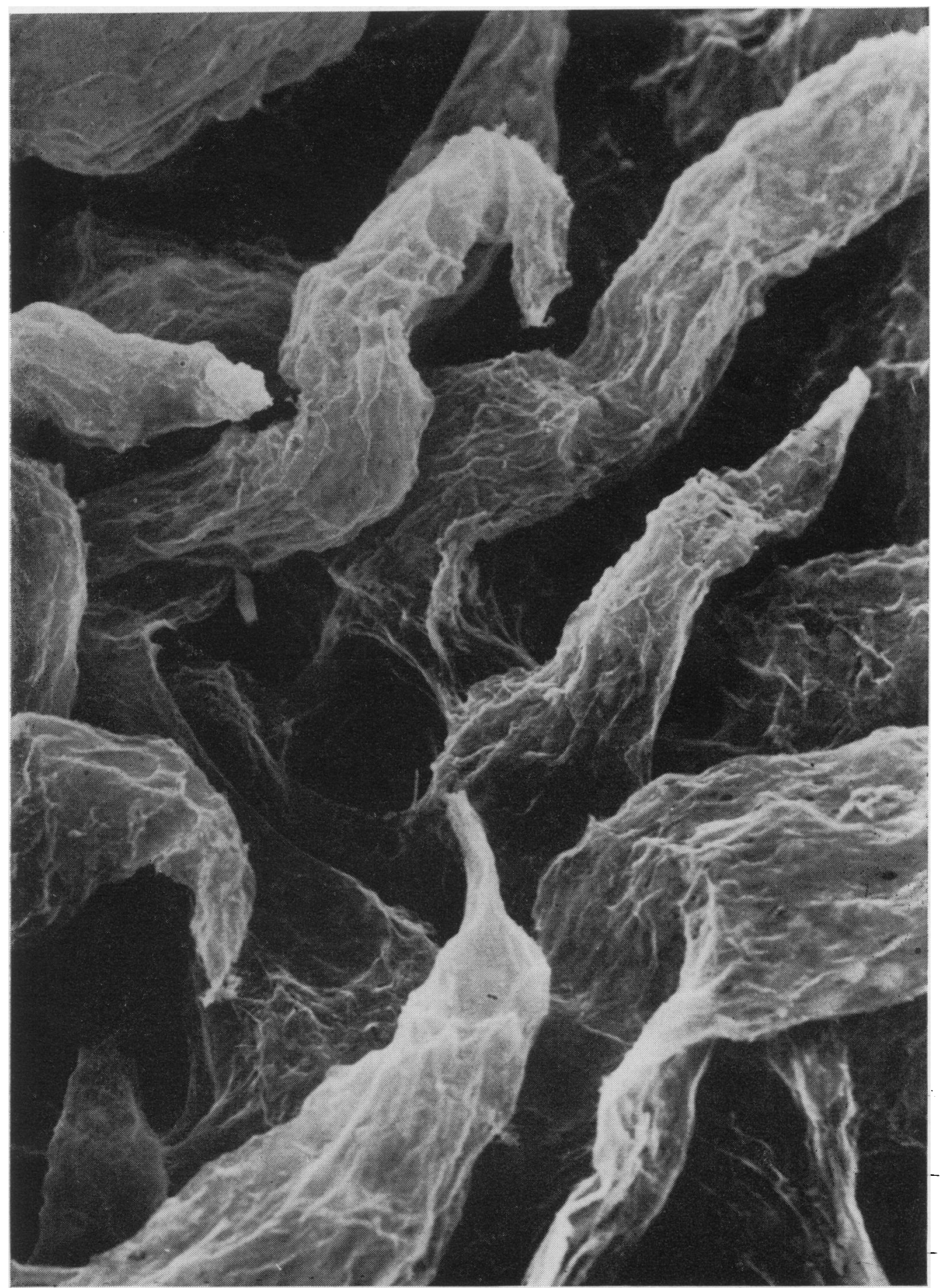

Fig. 9 Scanning electron micrograph of surviving villous cores from an autolysed necropsy specimen of jejunum from a human foetus. Predominantly finger-like villi are seen, although they are more distorted than in a fresh biopsy. $\times 500$. 


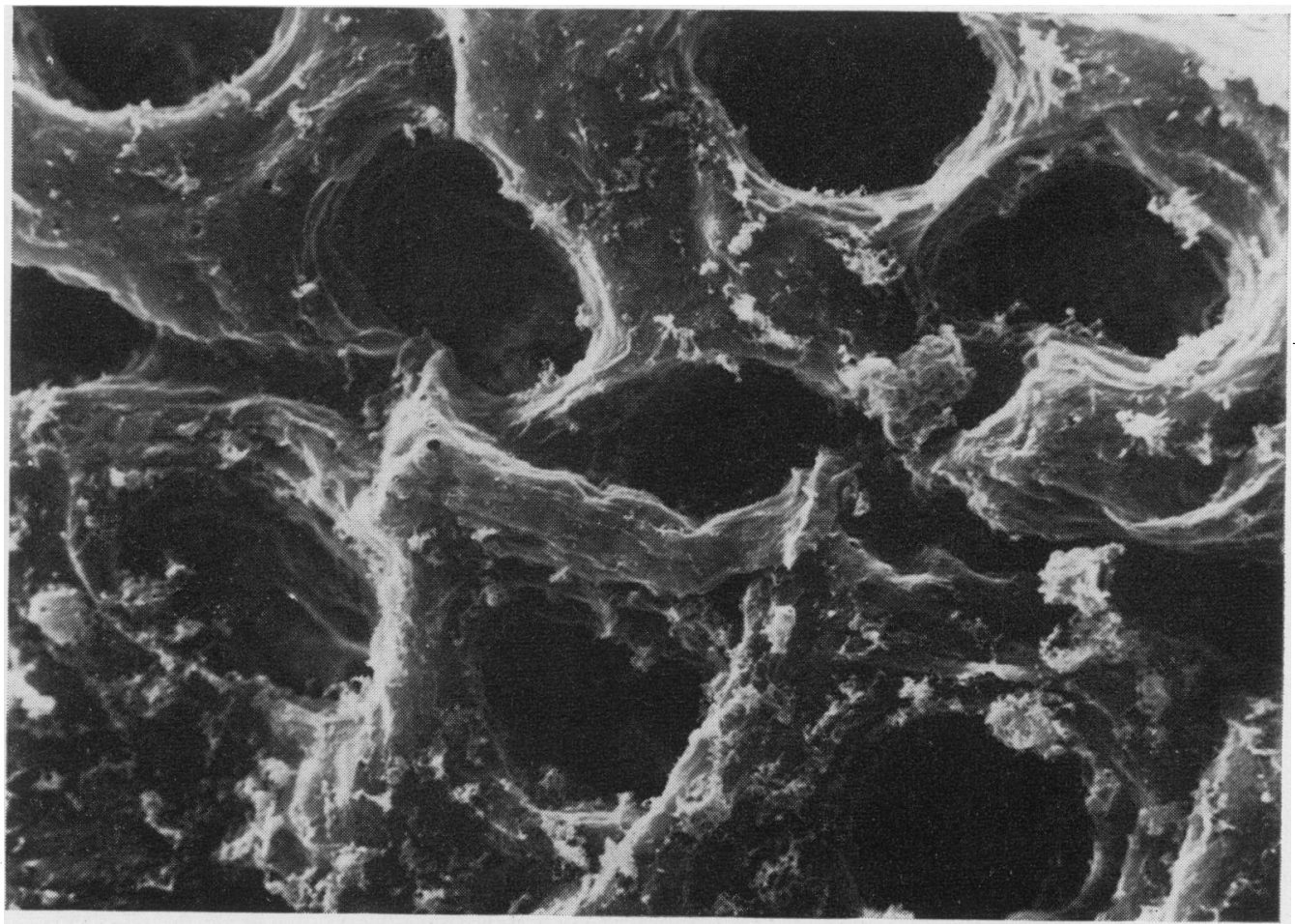

Fig. 10 Scanning electron micrograph of a flat mucosa. Autolysed necropsy specimen showing absence of villi and disproportionately wide crypt mouths, due to the loss of the lining epithelium. Moderate amounts of surface debris can be seen. $\times 325$.

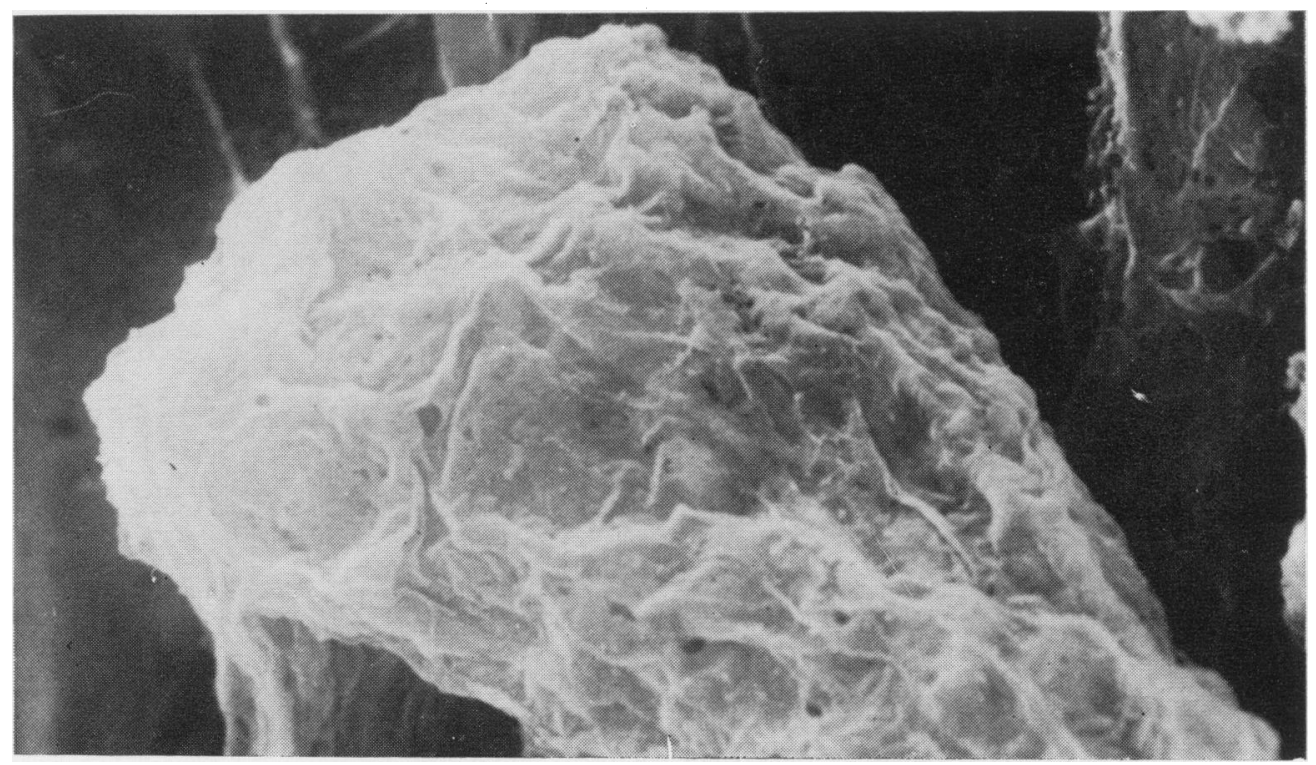

Fig. 11 Scanning electron micrograph of part of a villus from an autolysed necropsy specimen of jejunum from a human foetus. The irregular skeleton provided by the collagen framework may be seen, but the surface is surprisingly coherent. $\times 1,250$. 


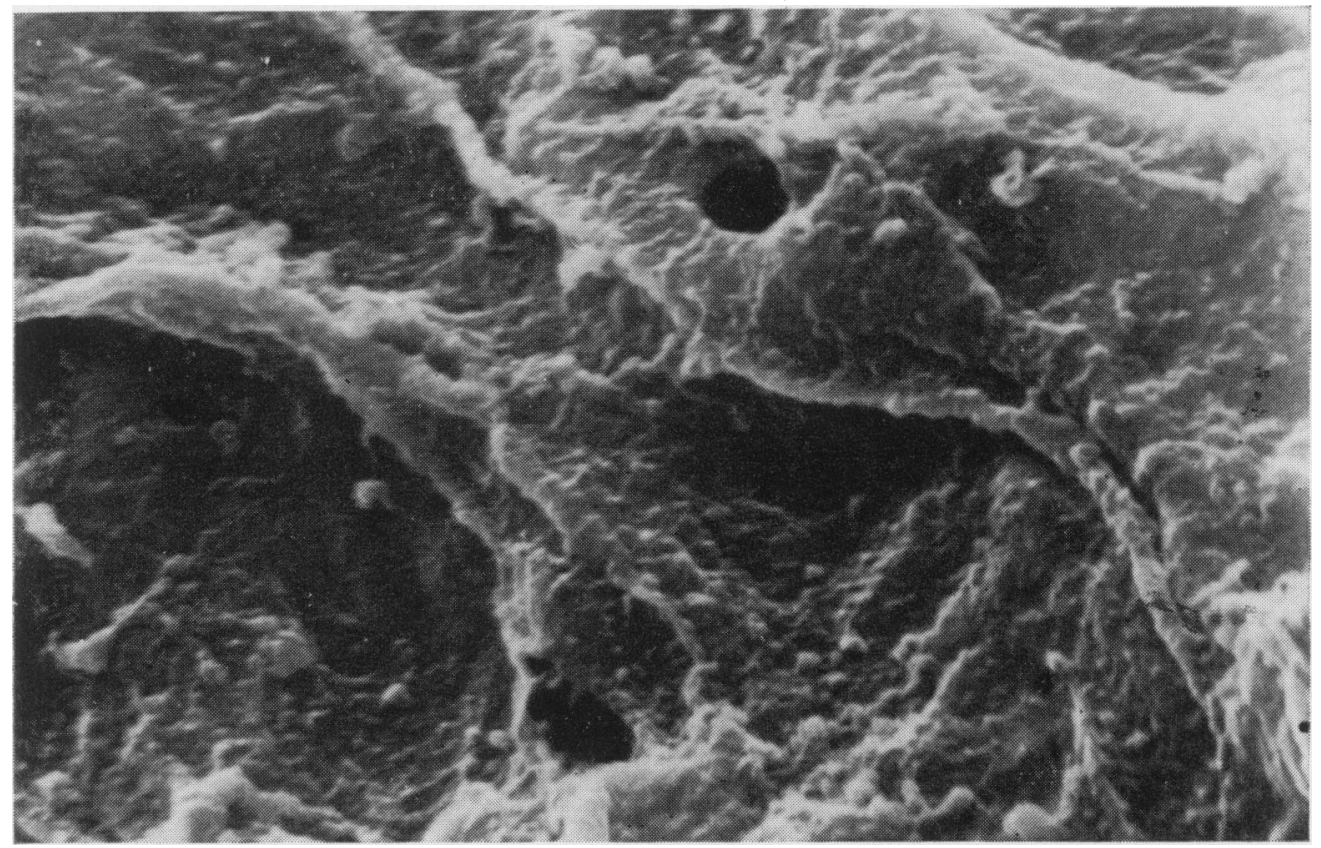

Fig. 12 Scanning electron micrograph of part of a villus from an autolysed necropsy specimen of jejunum from a human foetus. Two circular gaps are present in an otherwise coherent surface layer. The underlying collagen strands are sien. $\times 7,500$.

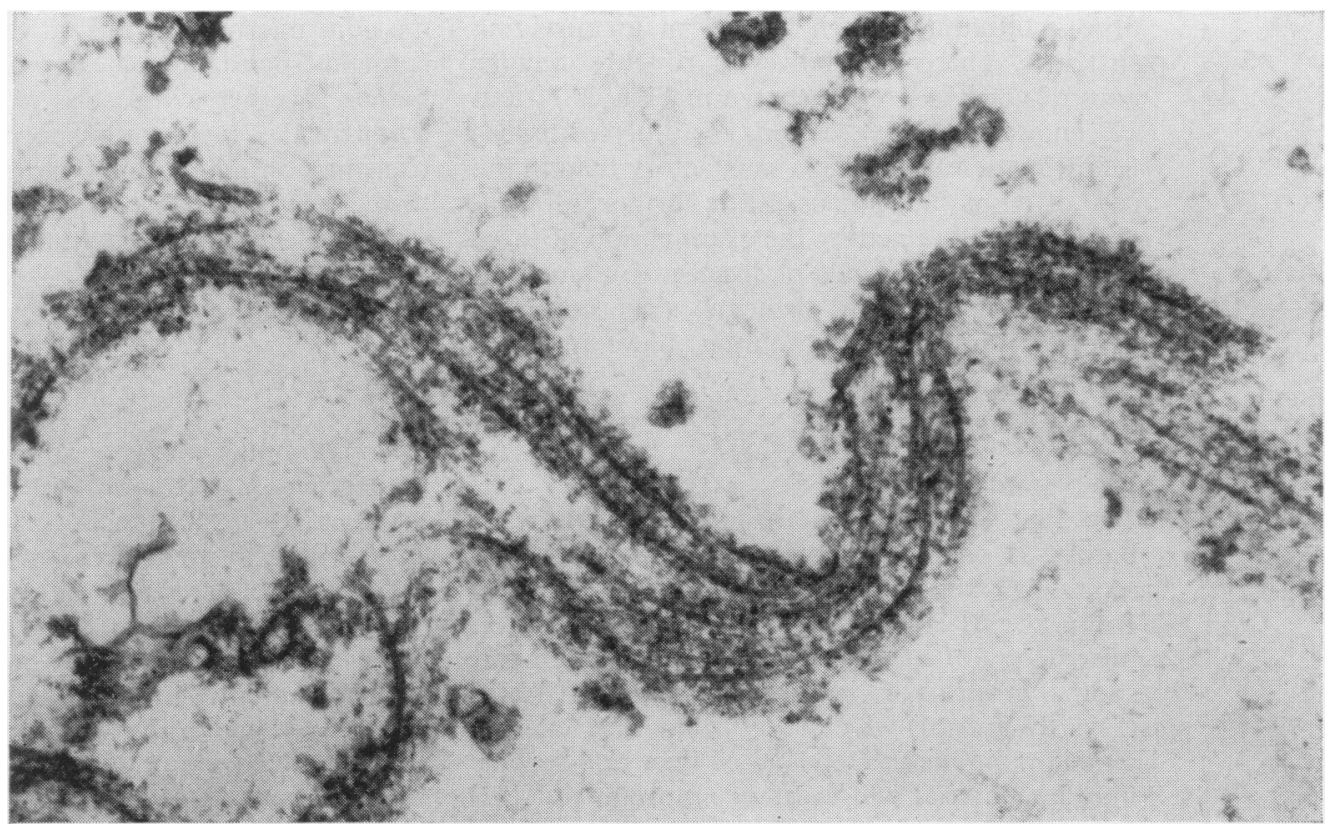

Fig. 13 Transmission electron micrograph of a thin section from the specimen illustrated in Figure 9. No cellular elements remain but the collagen framework is recognizable. Granular and amorphous debris is adherent to the fibre bundles, but there is no evidence of survival of a coherent basal lamina. $\times 35,000$. 


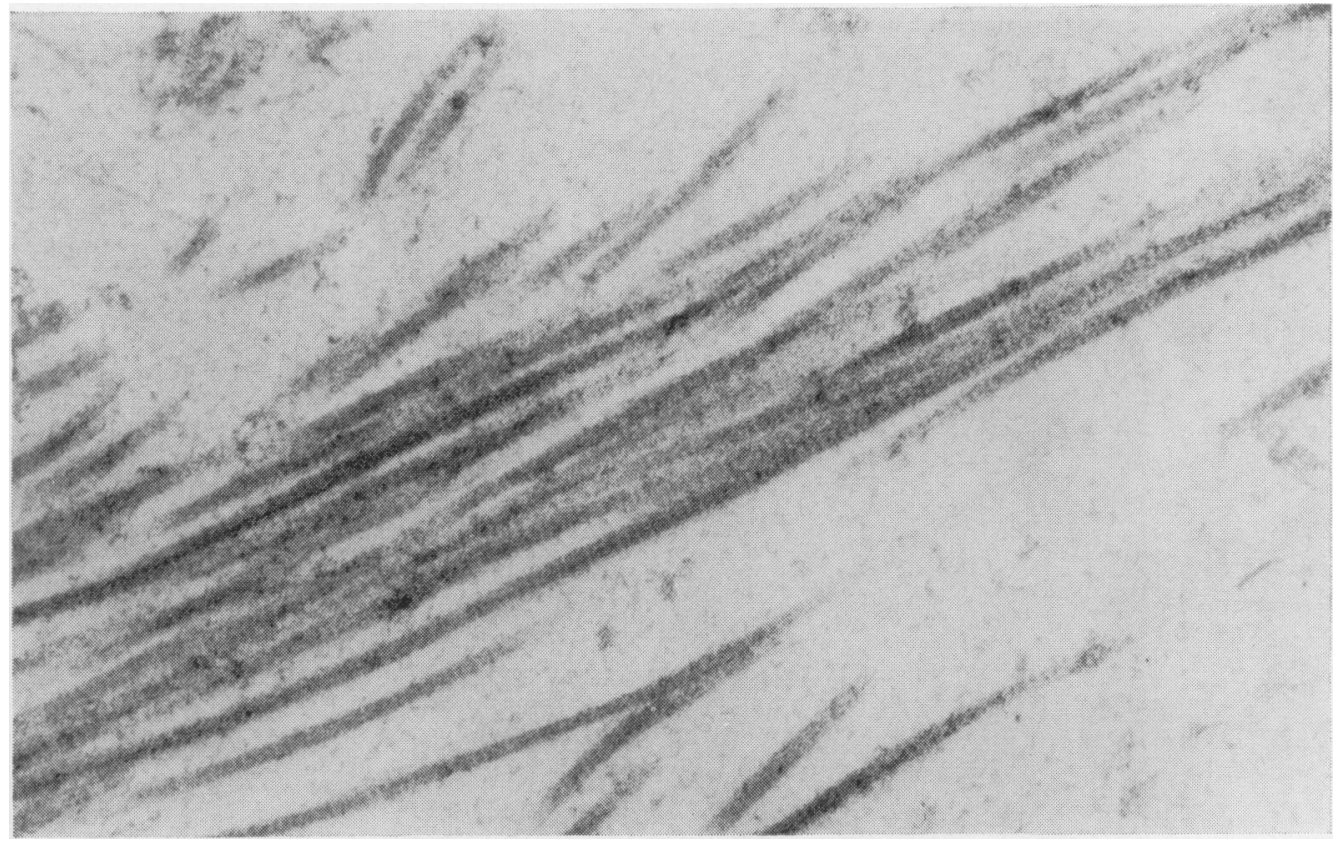

Fig. 14 The repeating periodicity of collagen is seen in these fibres from a similar specimen to that shown in Figure 13. $\times 62,000$.

continuous, as if covered by an external layer or 'basement membrane' spread over the collagen fibre scaffolding which is the main surviving structure. There are, however, roughly circular gaps of various sizes as shown in Figures 11 and 12. The study of $30 \mu$ sections of autolysed tissues following dewaxing is less satisfactory than in the case of biopsy specimens. Little further information can be obtained by the examination of these relatively flimsy strands of connective tissue, unsupported by cellular elements, since considerable distortion occurs during dewaxing and mounting.

\section{TRANSMISSION ELECTRON MICROSCOPY}

The fine structure of the intestinal mucosal biopsy as seen by transmission electron microscopy has been reviewed elsewhere (Trier and Rubin, 1965; Toner, 1968). Transmission electron microscopy of autolysed postmortem specimens shows groups of identifiable collagen fibres (Figs. 13 and 14) which still display their characteristic periodicity. Deposits of fine, non-specific fibrillar material and areas of amorphous debris accompany many of these groups of collagen fibres. Occasional cell remnants, such as ghost nuclei, are seen, and bacteria are identifiable. The surface imaged in the scanning microscope is the line of cleavage formed by the shedding of the epithelium. This represents the region of the histological 'basement membrane' of the villus, although there is no consistent evidence in transmission micrographs of a coherent surviving epithelial basal lamina. Remnants of the basal lamina together with debris of autolysis may account for the layer of non-specific debris noted on transmission microscopy, and may thus give rise to the relatively coherent envelope suggested in scanning electron micrographs.

\section{Discussion}

The scanning electron microscope combines moderately high resolution, $25 \mathrm{~m} \mu$, with great depth of focus, producing a three-dimensional effect in the image which is particularly suitable for studies of intestinal villi. The magnification range, from 20 times to 100,000 times, extends from the range of the dissecting light microscope to that of the transmission electron microscope. One disadvantage of the technique is the need for total dehydration of the specimen which may lead to tissue distortion and which rules out the examination of wet tissues. Despite possible sources of artefact, low magnification scanning micrographs of normal and autolysed mucosae were in strikingly close correspondence with the appearances on dissecting microscopy.

The technique of intestinal capsule biopsy has 
provided the means for recording qualitative changes of intestinal structure in disease (Rubin and Dobbins, 1965). The success of peroral biopsy has perhaps led to some neglect of alternative sources of tissue for investigation, even though some problems which concern the extent and significance of variations in villous form are not readily resolved by capsule biopsy. Such problems include the variations which occur from proximal to distal small intestine, and variations with age, from intrauterine to late adult life. In cases such as these, necropsy studies of the intestine are of particular value since much more material is available than is the case with capsule biopsies (Creamer and Leppard, 1965; Loehry and Creamer, 1966; Ferguson et al, 1969).

Despite the autolysis of the cellular components of the mucosa, the connective tissue fibre framework of the villi and the crypts survives to a considerable extent, preserving the form of the mucosa when examined by dissecting and transmission microscopy. These villous cores, however, lacking the moderate degree of rigidity imposed by an organized epithelium, lose much of their former mechanical stability and tend to fold, bend, and twist. The persistence of these outlines of villous architecture is related, in fine structural terms, to the survival of a framework of recognizable collagen fibres which show typical periodicity in transmission electron micrographs. Although, as shown by thin sections, the epithelial basal lamina does not survive intact, remnants of this structure associated with a loose surface coagulum of fine debris must account for the coherence of the villous surface as seen on scanning microscopy. The occasional gaps which can be seen seem more likely to be chance discontinuities produced during autolysis than meaningful structural features, but might represent discontinuities in the basal lamina and its collagen reinforcement caused by the transmigration of lymphocytes.

Significant variations in detail can probably result from the use of different techniques of preparing specimens for scanning electron microscopy. Microvilli, for example, were not clearly seen in whole mucosal specimens fixed as for transmission electron microscopy and air dried, but were easily shown in dewaxed $30 \mu$ sections and have been demonstrated in tissues freeze-dried after fixation (Marsh et al, 1968). Technique-dependent variations in detail in specimens may therefore require to be considered in interpretation. In general it is preferable to transfer to scanning electron microscopy those techniques known to give satisfactory preservation of fine structure at the level of transmission electron microscopy. An attempt must be made to retain the maximum comparability between these two techniques.

There are other problems of interpretation peculiar to scanning electron microscopy, such as the reliance mainly on surface-contour effects for image contrast. This raises particular difficulties of recognition and interpretation of details in high-resolution images. The relatively crude sectioning techniques used in this study are unable to show much significant intracellular detail and methods such as freeze fracturing will be more satisfactory for such work. Thick sections do, however, permit an unconventional view in depth of the larger structural features of the villi, including in particular the connective tissue components. Despite these problems, the limitations of the technique do not reduce the value of scanning electron microscopy in the study of the natural surface contours of the intestinal mucosa, whether obtained at biopsy or at necropsy.

The authors are grateful for research facilities provided by their respective departments.

\section{References}

Carr, K. E., and Toner, P. G. (1968). Scanning electron micro-

scopy of rat intestinal villi. Lancet, 2, 570-571.
Creamer, B., and Leppard, P. (1965). Post-mortem examination of a small intestine in the coeliac syndrome. Gut, 6,466 471 .

Demling, L., Becker, V., and Classen, M. (1969). Examinations of the mucosa of the small intestine with the scanning electron microscope. Digestion, 2, 51-60.

Ferguson, A., Maxwell, J. D., and Carr, K. E. (1969). Progressive changes in the small intestinal villous pattern with increasing length of gestation. J. Path., 99, 87-91."

Loehry, C. A., and Creamer, B. (1966). Post-mortem study of small intestinal mucosa. Brit. med. J., 1, 827-829.

Loehry, C. A., and Creamer, B. (1969). Three-dimensional structure of rat small intestinal mucosa related to mucosal dynamics. Gut, 10, 112-120.

Marsh, M. N., Swift, J. A., and Williams, E. D. (1968). Studies of small intestinal mucosa with the scanning electron microscope. Brit. med. J., 4, 95-96.

Rubin, C. E., and Dobbins, W. O. (1965). Peroral biopsy of the small intestine. A review of its diagnostic usefulness. Gastroenterology, 49, 676-697.

Shiner, M. (1956). Jejunal-biopsy tube. Lancet, 1, 85.

Shiner, M. (1957). Duodenal and jejunal biopsies. I. A discussion of the method, its difficulties and applications. Gastroenterology, 33, 64-70.

Toner, P. G. (1968). Cytology of intestinal epithelial cells. Int. Rev. Cytol., 24, 233-343.

Toner, P. G., and Carr, K. E. (1969). The use of scanning electron microscopy in the study of the intestinal villi. J. Path., 97,

Trier, J. S., and Rubin, C. E. (1965). Electron microscopy of the small intestine: a review. Gastroenterology, 49, 574-603. 